\title{
Political Connections and Enterprise Performance of Private Enterprises in China: Impacts and Mechanisms
}

\author{
Yang Wen ${ }^{1} \&$ Guo Feng ${ }^{2}$ \\ ${ }^{1}$ School of Economics, Central University of Finance and Economics, Beijing, China \\ ${ }^{2}$ School of Public Finance and Taxation, Central University of Finance and Economics, Beijing, China \\ Correspondence: Guo Feng, School of Public Finance and Taxation, Central University of Finance and \\ Economics, Beijing, China. Tel: 86-138-1038-7959. E-mail: yangwen@cufe.edu.cn
}

Received: August 21, 2020

Accepted: September 12, 2020

Online Published: September 22, 2020

doi:10.5539/ijef.v12n10p86

URL: https://doi.org/10.5539/ijef.v12n10p86

\begin{abstract}
Researchers have not come to an agreement on the impact of political connection on enterprise performance although this issue draws much attention. This paper attributes the above phenomenon to lack of precise classification of various political connection types in China. Based on existing literature, this paper divides political connections into current political connections and former political connections, and identifies their own hierarchy. Empirical study using Chinese Private Enterprise Survey data shows that different sorts of political connections all contribute to enterprise performance, and internal governance plays the mediating role. Overall, this paper may make marginal contributions to the study on the relationship between political connections and enterprise performance.
\end{abstract}

Keywords: private enterprises, political connections, internal governance, mechanism, enterprise performance

\section{Introduction}

Forty years after economic reform and open up, private enterprises benefit a lot from government supporting policies and become an important power in China. However, under the dual factors of China's specific cultural background and the economic system that still needs to be improved, the government who controls valuable and rare resources that enterprises need may allocate resources according to their relationship with the enterprises. Compared with state owned enterprises, private enterprises still confront with obvious or potential discriminations and obstacles. With imperfect formal system such as incomplete legal system, political connections can protect private enterprises from government infringement and industrial entry barriers, and enable enterprises to more easily obtain loans and preferential tax measures (Niessen \& Ruenzi, 2010; Li et al., 2013; Ba \& Zhu, 2017). On contrary, some studies argue that political connections can harm social efficiency when they intervene the credit, grants and capital markets, which creates extra social burdens (Boubakri et al., 2008; Liang \& Feng, 2010; He et al., 2013). And other scholars have got neutral opinions on this issue (Pan et al., 2005; Fan et al., 2007; Zhang \& Guo, 2010). In China, a society emphasizes social relationships or guanxi, what is the impact of political connections on China's private enterprise performance? This paper specially includes political connections classification and the mechanism in the analysis. Apart from current political connections like deputies to the National People's Congress (NPC) or members of China People's Political Consultative Conference (CPPCC) in existing papers, can former political connections such as government or state-owned enterprises working experiences affect enterprises performance?

Based on Chinese Private Enterprises Survey (2006-2014), this study tries to explain the impact, the path and the mechanism between political connections and private enterprise performance. The results imply that private enterprises that have political connections perform better than those who don't, and tend to get more complete internal governance structures.

\section{Literature Review and Hypothesis}

There are tons of research on China's private enterprise performance in the economic or management perspective, such as social capital (Yang, 2015), enterprise scale (Zhang, 2005), system environment (Yang, 2018), management strategy (Zhang, 2017), political connections (Luo \& Tang, 2009; Yu, 2010; He, 2019), environment regulations (Tisi, 2007; Ye \& Yang, 2020), management innovations (Yu, 2020), enterprise 
management (Gray, 2011; Qu et al., 2014). Political connection is one of the most important factors and critical to private enterprises' good performance in China (Faccio, 2006; Luo \& Huang, 2008; Hu, 2010; Tian \& Zhang, 2013).

In the context of transition economy in China, political connections mean a lot to private enterprises in that many restriction policies specifically target at private enterprises and impede their development. Zhou and Li (2003) and $\mathrm{Hu}$ (2006) argue that property protection scarcity, entry barriers and financial constraint are the main obstacles to private enterprises. Political connections help private enterprises to ease financial constraint (Yu, 2012), obtain financial benefit (Yu \& Pan, 2008; Li et al., 2008), get loan opportunities, apply government subsidy and support (Yu et al., 2010; Cai et al., 2011), lift investment efficiency (Chen \& Zhu, 2009; Chen et al., 2009; Cull et al., 2005), protect their property from government infringement (Pan, 2008), increase market value (Wu et al., 2008; Chen et al., 2011), etc. Based on the literature, this paper puts forward following hypotheses:

Hypothesis 1: It is better for private enterprises to maintain political connections.

It is necessary to identify whether different kinds of political connections impose various impacts on enterprise performance. Most scholars lay emphasis on current political connections, but this paper tries to classify political connections into former political connections and current political connections. We consider former political connections as work experience in government or state-owned enterprises that a private entrepreneur has; and current political connections as the position like deputy to National People's Congress or member of China People's Political Consultative Conference.

Since the reform and open policy, market economy in China has become a combination of old planning economy and market economy, which is also the feature of party and government institutions. Under these circumstances, private entrepreneurs may correlate themselves with former colleagues or friends to build political ties with the government and get desired resources. Meanwhile, entrepreneurs without former political connections would seek to take part in politic activities and link with the government. Therefore, private entrepreneurs are strongly motivated to be deputy to National People's Congress or member of China People's Political Consultative Conference. In this way, they can gain their reputation and social status, protect enterprise property, swap entry barriers and acquire more resources from government. This kind of political connections, which is established on statute and formal system, has stable systematic correlations with government and is better than former political connections based on personal social relationships. Upon above discussion, this paper proposes another hypothesis:

Hypothesis 2: Former and current political connections both contribute to enterprise performance, while current political connections do better than the other.

Existing literature illustrates that different kinds of political connections impose different effects on enterprise performance. Furthermore, can position or rank differentials affect enterprise performance variously? Faccio (2006) finds that the higher rank the political connections have, the more preferential tax policies and market occupation rate the enterprise enjoys. Faccio (2010) studies listed companies in 47 countries and finds that political connections can affect share price. Chinese scholars make similar conclusions on political connections in China as well. Liang and Feng (2010) holds that entrepreneurs can obtain more reputation and attention if he or she has higher political rank. Zhou (2009) deems that NPC or CPPCC members in different levels get divergent power to tackle financial issues. Hu (2006) finds that private entrepreneurs who have higher political positions have more chances to enter finance business. Zhang and Zhao (2014) finds that private entrepreneurs face different circumstances to accept government financial subsidies according to their positions in NCP or CPPCC. Therefore, we suppose that the tier or level of private enterprises' political connections may be a signal of enterprise strength and quality. Entrepreneurs with higher level political connections can influence government policy or get more resources to benefit the enterprise and promote enterprise performance. Based on that, this paper proposes the following hypothesis:

Hypothesis 3: Tiers of political connections positively affect enterprise performance. Compared with former political connections, current political connections affect more positively on enterprise performance.

Literature review reflects that political connections influence private enterprise performance in various paths. Theoretically, internal improvement and development of enterprises are critical to enterprise performance with regard to management structure and regulations. Good management is beneficial to enterprise performance; on contrary, an enterprise, which lacks fine internal governance, would lead to rent seeking related to somebody's own political connections. Galal and Mgeginsno's (1998) research about England, Chile, Malaysia and Mexico supports that management structure can enhance enterprise performance. Zhang and Zhang (2010) find that private listed companies perform better than state-owned companies in operation status, profitability, capital 
structure and market remarks. Meanwhile, some scholars find that political connected enterprises tend to slacken internal governance when they enjoy conveniences such as financing (Lei et al., 2009; Chaney, 2011).

Modern enterprise system in China was established with the help of government power. Internal governance of private enterprises shows different features from state-owned ones. On one hand, private entrepreneurs who have state-owned or collective-owned enterprise working experiences would try their best to take advantage of previous information channels, social network resources and internal governance experiences. Moreover, they also know well of the disadvantages of state-owned or collective-owned enterprises, such as inadequate motivations and insider control issues, which enables them to do optimal decisions. Private entrepreneurs with government-related working experiences are more familiar with the responsibilities, the regulations and work processes of party and government institutions. Balancing government-related resources advantages and enterprise management efficiency, they apply political networks to get more stable information and adjust management strategies dynamically. On the other hand, private entrepreneurs who are NPC or CPPCC members can motivate their employees to work hard and decrease agent problems. Additionally, by taking part in politics, private entrepreneurs voice their opinions and monitor on private economy issues, thus improve business environment to optimize internal performance. Based on that, this paper proposes the following hypothesis:

Hypothesis 4: Internal governance plays the mediating role between private entrepreneurs' political connections and enterprise performance.

Hypothesis 4a: Internal governance plays the mediating role between private entrepreneurs' different kinds of political connections and enterprise performance.

Hypothesis $4 \mathrm{~b}$ : Internal governance plays the mediating role between private entrepreneurs' different tiers of political connections and enterprise performance.

\section{Model and Method}

According to discussions above, a baseline estimation formula (1) is build to test the impacts of private entrepreneurs' political connections on enterprise performance. In formula (1), return on assets (ROA) represents enterprise performance, the dependent variable, and X represents independent variables, including total political connections (pcon), former political connections (fpoc), current political connections (cpoc) and the tier of political connections. Among them, total political connections, former political connections and current political connections are 0-1 variables. The tier of political connections are identifies as follows: current political connections such as the tier of NPC (repp) and the tier of CPPCC (cppc) are 0-5 variables, the position in NPC (rpos) and the position in CPPCC (cppm) are 0-3 variables; former political connections like the position in government institutions is a 0-4 variable and posts in state-owned enterprises is a 0-5 variable. Control variables of enterprises and entrepreneurs include enterprise scale, enterprise age, assets liabilities ratio, enterprise assets scale, industry status, entrepreneur gender, entrepreneur age, entrepreneur education background. Besides, province effects, year effects and industry effects are also controlled.

$$
\begin{aligned}
& \text { ROA }_{i}=\alpha+\beta_{1} x_{i}+\beta_{2} \text { empl }_{i}+\beta_{3} \text { agec }_{i}+\beta_{4} \text { alra }_{i}+\beta_{5} \text { ltass }_{i}+\beta_{6} \text { ind }_{i} \\
& +\beta_{7} \text { gend }_{i}+\beta_{8} \text { age }_{i}+\beta_{9} \text { edu }_{i}+\text { year }+ \text { prov }+ \text { ind }+\varepsilon_{i}
\end{aligned}
$$

The baseline regression results are as follows:

\begin{tabular}{|c|c|c|c|}
\hline & Model (1) & Model (2) & Model (3) \\
\hline pcon & $\begin{array}{c}5.531 * * * \\
(3.76)\end{array}$ & & \\
\hline fpoc & & $\begin{array}{c}4.638 * * * \\
(4.00)\end{array}$ & \\
\hline срос & & & $\begin{array}{c}5.676^{* * *} \\
(4.81)\end{array}$ \\
\hline empl & $\begin{array}{c}12.369 * * * \\
(17.09)\end{array}$ & $\begin{array}{c}12.518 * * * \\
(17.32)\end{array}$ & $\begin{array}{c}12.110^{* * * *} \\
(16.92)\end{array}$ \\
\hline agec & $\begin{array}{l}0.164 \\
(1.37)\end{array}$ & $\begin{array}{l}0.210^{*} \\
(1.74)\end{array}$ & $\begin{array}{l}0.134 \\
(1.12)\end{array}$ \\
\hline alra & $\begin{array}{c}-0.078 * * * \\
(-4.06)\end{array}$ & $\begin{array}{c}-0.078 * * * \\
(-4.07)\end{array}$ & $\begin{array}{c}-0.076 * * * \\
(-3.97)\end{array}$ \\
\hline ltass & $\begin{array}{c}-13.664 * * * \\
(-21.86)\end{array}$ & $\begin{array}{c}-13.593 * * * \\
(-21.74)\end{array}$ & $\begin{array}{c}-13.811 * * * \\
(-21.83)\end{array}$ \\
\hline
\end{tabular}

Table 1. Entrepreneurs' political connections and enterprise performance (baseline regression) 


\begin{tabular}{cccc}
\hline indc & $0.639 * * *$ & $0.639 * * *$ & $0.642 * * *$ \\
& $(3.27)$ & $(3.28)$ & $(3.25)$ \\
gend & -0.149 & -0.008 & -0.078 \\
& $(-0.09)$ & $(-0.00)$ & $(-0.04)$ \\
age & -0.078 & -0.091 & -0.046 \\
& $(-1.10)$ & $(-1.28)$ & $(-0.66)$ \\
edu & $1.592^{* * *}$ & $1.548 * * *$ & $1.708^{* * *}$ \\
& $(3.06)$ & $(2.95)$ & $(3.28)$ \\
_cons & $47.819 * * *$ & $48.737 * * *$ & $50.089 * * *$ \\
province & $(8.77)$ & $(8.96)$ & $(9.22)$ \\
industry & Control & control & control \\
year & Control & control & control \\
$\mathrm{N}$ & Control & control & 7872.000 \\
r2 & 7872.000 & 7872.000 & 0.174 \\
\hline
\end{tabular}

Note. $\mathrm{t}$ statistics in parentheses, $* \mathrm{p}<0.1, * * \mathrm{p}<0.05$, *** $\mathrm{p}<0.01$.

Table 1 illustrates the impacts of various political connections on private enterprise performance, including total political connections (Model (1)), current political connections (Model (2)) and former political connections (Model (3)). Empirical results show that all the above connections boost private enterprise performance. Enterprises which have political connections perform $5.531 \%$ better than those don't have. And among private enterprises with political connections, enterprises with current political connections performs $1.038 \%$ better than those having former political connections. Hypothesis 1 and hypothesis 2 are proven.

Table 2. The impacts of different tiers of political connections on enterprise performance

\begin{tabular}{|c|c|c|c|c|c|c|}
\hline & Model (4) & Model (5) & Model (6) & Model (7) & Model (8) & Model (9) \\
\hline repp & $\begin{array}{c}2.103 * * * \\
(3.97)\end{array}$ & & & & & \\
\hline rpos & & $\begin{array}{c}6.679 * * * \\
(2.68)\end{array}$ & & & & \\
\hline сppc & & & $\begin{array}{c}1.189 * * \\
(2.43)\end{array}$ & & & \\
\hline cppm & & & & $\begin{array}{c}3.445^{* *} \\
(2.25)\end{array}$ & & \\
\hline parg & & & & & $\begin{array}{c}1.999 * * \\
(2.30)\end{array}$ & \\
\hline esta & & & & & & $\begin{array}{c}1.146^{* * * *} \\
(4.30)\end{array}$ \\
\hline empl & $\begin{array}{c}12.181 \text { *** } \\
(17.11)\end{array}$ & $\begin{array}{c}12.473^{* * * *} \\
(17.28)\end{array}$ & $\begin{array}{c}12.492 * * * \\
(17.27)\end{array}$ & $\begin{array}{c}12.518 * * * \\
(17.40)\end{array}$ & $\begin{array}{c}12.547 * * * \\
(17.38)\end{array}$ & $\begin{array}{c}12.501 \text { *** } \\
(17.28)\end{array}$ \\
\hline agec & $\begin{array}{l}0.185 \\
(1.54)\end{array}$ & $\begin{array}{l}0.196 \\
(1.63)\end{array}$ & $\begin{array}{l}0.165 \\
(1.36)\end{array}$ & $\begin{array}{l}0.184 \\
(1.52)\end{array}$ & $\begin{array}{c}0.213^{*} \\
(1.76)\end{array}$ & $\begin{array}{c}0.223^{*} \\
(1.85)\end{array}$ \\
\hline alra & $\begin{array}{c}-0.077 * * * \\
(-4.00)\end{array}$ & $\begin{array}{c}-0.077 \text { **** } \\
(-3.98)\end{array}$ & $\begin{array}{c}-0.075 * * * \\
(-3.91)\end{array}$ & $\begin{array}{c}-0.076 * * * \\
(-3.95)\end{array}$ & $\begin{array}{c}-0.076 * * * \\
(-3.96)\end{array}$ & $\begin{array}{c}-0.079 * * * \\
(-4.07)\end{array}$ \\
\hline ltass & $\begin{array}{c}-13.736 * * * \\
(-21.70)\end{array}$ & $\begin{array}{c}-13.633 * * * \\
(-21.71)\end{array}$ & $\begin{array}{c}-13.654 * * * \\
(-21.76)\end{array}$ & $\begin{array}{c}-13.618^{* * *} \\
(-21.68)\end{array}$ & $\begin{array}{c}-13.596 * * * \\
(-21.71)\end{array}$ & $\begin{array}{c}-13.593 * * * \\
(-21.74)\end{array}$ \\
\hline indc & $\begin{array}{c}0.634 * * * \\
(3.23)\end{array}$ & $\begin{array}{c}0.642 * * * \\
(3.28)\end{array}$ & $\begin{array}{c}0.640 * * * \\
(3.26)\end{array}$ & $\begin{array}{c}0.641 * * * \\
(3.28)\end{array}$ & $\begin{array}{c}0.642 * * * \\
(3.29)\end{array}$ & $\begin{array}{c}0.639 * * * \\
(3.27)\end{array}$ \\
\hline gend & $\begin{array}{l}0.287 \\
(0.16)\end{array}$ & $\begin{array}{l}0.121 \\
(0.07)\end{array}$ & $\begin{array}{l}-0.059 \\
(-0.03)\end{array}$ & $\begin{array}{l}-0.035 \\
(-0.02)\end{array}$ & $\begin{array}{l}-0.065 \\
(-0.04)\end{array}$ & $\begin{array}{l}-0.325 \\
(-0.19)\end{array}$ \\
\hline age & $\begin{array}{l}-0.051 \\
(-0.73)\end{array}$ & $\begin{array}{l}-0.037 \\
(-0.54)\end{array}$ & $\begin{array}{l}-0.032 \\
(-0.46)\end{array}$ & $\begin{array}{l}-0.038 \\
(-0.54)\end{array}$ & $\begin{array}{l}-0.054 \\
(-0.77)\end{array}$ & $\begin{array}{l}-0.102 \\
(-1.42)\end{array}$ \\
\hline edu & $\begin{array}{c}1.743 * * * \\
(3.34)\end{array}$ & $\begin{array}{c}1.770^{* * * *} \\
(3.39)\end{array}$ & $\begin{array}{c}1.736 * * * \\
(3.33)\end{array}$ & $\begin{array}{c}1.743 * * * \\
(3.34)\end{array}$ & $\begin{array}{c}1.673^{* * *} \\
(3.19)\end{array}$ & $\begin{array}{c}1.568 * * * \\
(2.99)\end{array}$ \\
\hline _cons & $\begin{array}{c}51.002 * * * \\
(9.25)\end{array}$ & $\begin{array}{c}49.070^{* * * *} \\
(9.01)\end{array}$ & $\begin{array}{c}48.589 * * * \\
(8.94)\end{array}$ & $\begin{array}{c}48.721 * * * \\
(8.97)\end{array}$ & $\begin{array}{c}48.923 * * * \\
(8.94)\end{array}$ & $\begin{array}{c}50.475 * * * \\
(9.27)\end{array}$ \\
\hline province & control & Control & control & control & control & control \\
\hline industry & control & Control & control & control & control & control \\
\hline year & control & Control & control & control & control & control \\
\hline $\mathrm{N}$ & 7872.000 & 7872.000 & 7872.000 & 7872.000 & 7872.000 & 7872.000 \\
\hline $\mathrm{r} 2$ & 0.174 & 0.173 & 0.172 & 0.172 & 0.172 & 0.174 \\
\hline
\end{tabular}

Note. $\mathrm{t}$ statistics in parentheses, $* \mathrm{p}<0.1, * * \mathrm{p}<0.05$, *** $\mathrm{p}<0.01$. 
In Table 2, independent variables of Model (4)- Model (9) are respectively the tier of NPC, the position in NPC, the tier of CPPCC, the position in CPPCC, the position in government institutions and the position in state-owned or collective-owned enterprises, while their control variables are the same. Regression results shows that all the above independent variables are positive to enterprise performance and the higher the position or the tier, the more positive the effects on enterprise performance would be. Among current political connections, the tier of NPC and the position of NPC impose more effects on enterprise performance than the tier of CPPCC and the position of CPPCC. Therefore, hypothesis 3 is verified.

Due to potential biases of selection and omitted variables in OLS regression, the results can be not consistent and stable. This section takes propensity score matching and proxy variable estimation (shareholding ratio of the entrepreneur and his/her family is the proxy variable to identify entrepreneur's personal capacity) to test endogeneity issues. The two tests further agree with the results of baseline regression (restricted to space, no more details), which strongly supports hypothesis 1-3 in this paper.

\section{Mechanism and Empirical Results}

The analysis above has shown the effects of private entrepreneurs' political connections on enterprise performance, and this section is going to discuss the role of internal governance in the relationship between the connections and the performance. Referring to the method to design management structure index by Gompers et al. (2003), this paper selects 10 questions in the questionnaire linking with internal governance to build the variables and all of them are binary variables. The enterprise will get 1 score when its internal governance satisfies with one of the questions, thus the score of internal governance maximizes to 10 . With reference to mediating effects tests proposed by Wen et al. (2014), this paper sets the following three-step regression formulas:

$$
\begin{gathered}
\text { ROA }_{i}=\alpha+\beta_{1} x_{i}+\beta_{2} \text { empl }_{i}+\beta_{3} \text { agec }_{i}+\beta_{4} \text { alra }_{i}+\beta_{5} \text { ltass }_{i}+\beta_{6} \text { indc }_{i} \\
+\beta_{7} \text { gend }_{i}+\beta_{8} \text { age }_{i}+\beta_{9} \text { edu }_{i}+\beta_{10} \text { ownp }_{i}+\text { year }+ \text { prov } \text { ind }+\varepsilon_{i} \\
M_{i}=\alpha+\beta_{1} x_{i}+\beta_{2} \text { empl }_{i}+\beta_{3} \text { agec }_{i}+\beta_{4} \text { alra }_{i}+\beta_{5} \text { ltass }_{i}+\beta_{6} i n d c_{i} \\
+\beta_{7} \text { gend }_{i}+\beta_{8} \text { age }_{i}+\beta_{9} \text { edu }_{i}+\beta_{10} \text { ownp }_{i}+\text { year }+ \text { prov }+ \text { ind }+\varepsilon_{i} \\
\text { ROA }_{i}=\alpha+\beta_{1} x_{i}+\gamma M_{i}+\beta_{2} \text { empl }_{i}+\beta_{3} \text { agec }_{i}+\beta_{4} \text { alra }_{i}+\beta_{5} \text { ltass }_{i}+\beta_{6} \text { indc }_{i} \\
+\beta_{7} \text { gend }_{i}+\beta_{8} \text { age }_{i}+\beta_{9} \text { edu }_{i}+\beta_{10} \text { ownp }_{i}+\text { year }+ \text { prov }+ \text { ind }+\varepsilon_{i}
\end{gathered}
$$

Table 3, Table 4 and Table 5 respectively reports the empirical results of the impacts of total political connections, different kinds of political connections and different tiers of political connections on private enterprise performance through enterprise internal governance.

Table 3. Impact mechanism of total political connections (enterprise internal governance)

\begin{tabular}{cccc}
\hline variables & ROA & admt & ROA \\
\hline pcon & $5.517 * * *$ & $0.397 * * *$ & $(3.32)$ \\
& $(3.75)$ & $(7.77)$ & $1.545^{* * *}$ \\
admt & & & $(4.85)$ \\
& $12.312^{* * *}$ & $0.525 * * *$ & $11.501^{* * *}$ \\
empl & $(17.06)$ & $(25.72)$ & $(16.06)$ \\
& $0.214^{*}$ & $-0.009 *$ & $0.227 *$ \\
agec & $(1.73)$ & $(-1.89)$ & $(1.85)$ \\
& $-0.080^{* * *}$ & $0.003 * * *$ & $-0.085^{* * *}$ \\
alra & $(-4.15)$ & $(3.88)$ & $(-4.41)$ \\
& $-13.733^{* * *}$ & $0.206 * * *$ & $-14.051^{* * *}$ \\
ltass & $(-21.86)$ & $(13.55)$ & $(-21.87)$ \\
& $0.633^{* * *}$ & $0.013 * * *$ & $0.613 * * *$ \\
indc & $(3.27)$ & $(3.72)$ & $(3.13)$ \\
& -0.074 & 0.053 & -0.156 \\
gend & $(-0.04)$ & $(0.87)$ & $(-0.09)$ \\
& -0.088 & $0.009 * * *$ & -0.102 \\
age & $(-1.25)$ & $(3.41)$ & $(-1.44)$ \\
& $1.538^{* * *}$ & $0.090^{* * *}$ & $1.399 * * *$ \\
edu & $(2.96)$ & $(4.81)$ & $(2.71)$ \\
\end{tabular}




\begin{tabular}{cccc}
\hline ownp & $-0.053^{* * *}$ & $-0.015^{* * *}$ & -0.029 \\
& $(-2.69)$ & $(-21.28)$ & $(-1.43)$ \\
_cons & $52.229^{* * *}$ & $0.942^{* * *}$ & $50.773^{* * *}$ \\
& $(8.89)$ & $(4.35)$ & $(8.63)$ \\
province & control & control & control \\
industry & control & control & control \\
year & control & control & control \\
$\mathrm{N}$ & 7872.000 & 7872.000 & 7872.000 \\
$\mathrm{r} 2$ & 0.174 & 0.373 & 0.177 \\
Sobel Z & & $4.116^{* * *}$ & \\
\hline
\end{tabular}

Note. $\mathrm{t}$ statistics in parentheses, ${ }^{*} \mathrm{p}<0.1, * * \mathrm{p}<0.05, * * * \mathrm{p}<0.01$.

Table 3 displays the empirical results of how total political connections affect private enterprise performance through internal governance. The first column shows total political connections positively affect private enterprise performance $(\beta 1=5.517, \mathrm{p}<0.01)$. The second column illustrates that total political connections have positive effects on enterprise internal governance $(\beta 1=0.397, p<0.01)$. And coefficient $r$ is significant in the third column $(\gamma=1.545, \mathrm{p}<0.01)$, which means enterprise internal governance is the mediating factor to influence enterprise performance. Coefficient $\beta 1$ is 4.904 and positive, and $\beta 1$ (formula (3)) $\gamma^{\prime}$ (formula (4)) and $\beta 1$ (formula (2)) are both positive, which implies partial mediating effects and the mediating effects account for $11.1 \%$ $(=0.397 * 1.545 / 5.517)$ in total effects. Hypothesis 4 is thus proven.

Table 4. Impact mechanism of former and current political connections (enterprise internal governance)

\begin{tabular}{|c|c|c|c|c|c|c|}
\hline \multirow{2}{*}{ variables } & \multicolumn{3}{|c|}{ Former political connections } & \multicolumn{3}{|c|}{ Current political connections } \\
\hline & ROA & admt & ROA & ROA & admt & ROA \\
\hline \multirow[t]{2}{*}{ fpoc } & $4.398 * * *$ & $0.232 * * *$ & $4.034 * * *$ & & & \\
\hline & $(3.75)$ & $(5.37)$ & $(3.42)$ & & & \\
\hline \multirow[t]{2}{*}{ admt } & & & $1.570^{* * *}$ & & & $1.486 * * *$ \\
\hline & & & $(4.92)$ & & & $(4.69)$ \\
\hline \multirow[t]{2}{*}{ cpoc } & & & & $6.035^{* * *}$ & $0.416^{* * *}$ & $5.417 * * *$ \\
\hline & & & & $(5.08)$ & $(9.34)$ & $(4.57)$ \\
\hline \multirow[t]{2}{*}{ empl } & $12.470^{* * *}$ & $0.537 * * *$ & $11.626 * * *$ & $12.013 * * *$ & $0.505^{* * *}$ & $11.263 * * *$ \\
\hline & $(17.30)$ & $(26.43)$ & $(16.26)$ & $(16.85)$ & $(24.53)$ & $(15.85)$ \\
\hline \multirow[t]{2}{*}{ agec } & $0.253^{* *}$ & -0.006 & $0.263 * *$ & 0.189 & $-0.010^{* *}$ & $0.204 *$ \\
\hline & $(2.05)$ & $(-1.29)$ & $(2.13)$ & $(1.53)$ & $(-2.25)$ & $(1.66)$ \\
\hline \multirow[t]{2}{*}{ alra } & $-0.080 * * *$ & $0.003 * * *$ & $-0.085 * * *$ & $-0.078 * * *$ & $0.003 * * *$ & $-0.083^{* * *}$ \\
\hline & $(-4.14)$ & (3.94) & $(-4.42)$ & $(-4.07)$ & $(4.06)$ & $(-4.34)$ \\
\hline \multirow[t]{2}{*}{ ltass } & $-13.653 * * *$ & $0.212 * * *$ & $-13.987 * * *$ & $-13.907 * * *$ & $0.195 * * *$ & $-14.196^{* * * *}$ \\
\hline & $(-21.73)$ & (13.92) & $(-21.75)$ & $(-21.84)$ & $(12.71)$ & $(-21.86)$ \\
\hline \multirow[t]{2}{*}{ indc } & $0.634 * * *$ & $0.013 * * *$ & $0.614 * * *$ & $0.635 * * *$ & $0.013^{* * *}$ & $0.615^{* * *}$ \\
\hline & $(3.27)$ & (3.74) & (3.13) & (3.24) & (3.78) & $(3.11)$ \\
\hline \multirow[t]{2}{*}{ gend } & 0.060 & 0.063 & -0.039 & 0.005 & 0.059 & -0.083 \\
\hline & $(0.03)$ & (1.03) & $(-0.02)$ & 0.00 & $(0.96)$ & $(-0.05)$ \\
\hline \multirow[t]{2}{*}{ age } & -0.098 & $0.009 * * *$ & -0.112 & -0.059 & $0.011^{* * *}$ & -0.075 \\
\hline & $(-1.37)$ & (3.49) & $(-1.58)$ & $(-0.84)$ & $(4.27)$ & $(-1.07)$ \\
\hline \multirow[t]{2}{*}{ edu } & $1.511^{* * *}$ & $0.092 * * *$ & $1.367 * * *$ & $1.641 * * *$ & $0.098 * * *$ & $1.496^{* * * *}$ \\
\hline & (2.88) & $(4.88)$ & $(2.63)$ & (3.16) & $(5.25)$ & $(2.90)$ \\
\hline \multirow[t]{2}{*}{ ownp } & $-0.047 * *$ & $-0.015^{* * *}$ & -0.023 & $-0.062 * * *$ & $-0.016^{* * *}$ & $-0.038^{*}$ \\
\hline & $(-2.37)$ & $(-20.78)$ & $(-1.15)$ & $(-3.14)$ & $(-22.15)$ & $(-1.87)$ \\
\hline \multirow[t]{2}{*}{ _cons } & $52.650^{* * *}$ & $0.974 * * *$ & $51.120^{* * *}$ & $55.374 * * *$ & $1.161^{* * *}$ & $53.650 * * *$ \\
\hline & (8.98) & (4.49) & (8.71) & $(9.45)$ & $(5.33)$ & $(9.14)$ \\
\hline province & control & control & control & control & control & control \\
\hline industry & control & control & control & control & control & control \\
\hline year & control & control & control & control & control & control \\
\hline $\mathrm{N}$ & 7872.000 & 7872.000 & 7872.000 & 7872.000 & 7872.000 & 7872.000 \\
\hline $\mathrm{r} 2$ & 0.174 & 0.371 & 0.177 & 0.175 & 0.376 & 0.178 \\
\hline Sobel Z & & $3.629 * * *$ & & & $4.192^{* * * *}$ & \\
\hline
\end{tabular}

Note. $\mathrm{t}$ statistics in parentheses, $* \mathrm{p}<0.1, * * \mathrm{p}<0.05, * * * \mathrm{p}<0.01$. 
Table 4 exhibits the empirical results of the impacts of various kinds of political connections on private enterprise performance. Concerning former political connections, the first column shows former political connections impose positive effects on private enterprise performance $(\beta 1=4.398, p<0.01)$. The second column displays that former political connections affect enterprise internal governance positively $(\beta 1=0.232, p<0.01)$. At the same time, coefficient $\gamma$ is significant $(\gamma=1.57, \mathrm{p}<0.01)$ in the third column, which means that enterprise internal governance is the mediating factor to promote enterprise performance. Coefficient $\beta 1$ in the third column is positive 4.034, and coefficients $\beta 1$ (formula (3))* $\gamma$ (formula (4)) and $\beta 1$ (formula (2)) are both positive, which implies partial mediating effects and the mediating effects occupy $8.3 \%(=0.232 * 1.57 / 4.398)$ in total effects. For current political connections, the fourth column shows political connections are positive to private enterprise performance $(\beta 1=6.035, \mathrm{p}<0.01)$. The fifth column displays that current political connections affect enterprise internal governance positively $(\beta 1=0.416, p<0.01)$. Meanwhile, coefficient $\gamma$ is significant $(\gamma=1.486$, $\mathrm{p}<0.01)$ in the sixth column, which implies that enterprise internal governance is the mediating factor to promote enterprise performance. Coefficient $\beta 1$ in the sixth column is positive 4.034, and $\beta 1$ (formula (3))* $\gamma$ (formula (4)) and $\beta 1$ (formula (2)) are both positive, which means partial mediating effects and the mediating effects contribute $10.2 \%(=0.416 * 1.486 / 6.035)$ to total effects. Overall, internal governance can be a mediating factor between political connections and enterprise performance. Hypothesis $4 \mathrm{a}$ is therefore proven.

Table 5. Impact mechanism of the tire of NPC and the position in NPC

\begin{tabular}{|c|c|c|c|c|c|c|}
\hline \multirow{2}{*}{ variables } & \multicolumn{3}{|c|}{ The tier of NPC } & \multicolumn{3}{|c|}{ The position in NPC } \\
\hline & ROA & jcb & ROA & ROA & $\mathrm{jcb}$ & ROA \\
\hline \multirow[t]{2}{*}{ repp } & $2.165 * * *$ & $0.187 * * *$ & $1.881 * * *$ & & & \\
\hline & $(4.09)$ & $(9.29)$ & $(3.55)$ & & & \\
\hline \multirow[t]{2}{*}{ admt } & & & $1.515 * * *$ & & & $1.594 * * *$ \\
\hline & & & $(4.76)$ & & & $(5.00)$ \\
\hline \multirow[t]{2}{*}{ rpos } & & & & $6.665^{* * *}$ & $0.419 * * *$ & $5.998 * *$ \\
\hline & & & & $(2.68)$ & $(4.96)$ & $(2.40)$ \\
\hline \multirow[t]{2}{*}{ empl } & $12.109 * * *$ & $0.504 * * *$ & $11.344 * * *$ & $12.416^{* * *}$ & $0.533 * * *$ & $11.566 * * *$ \\
\hline & (17.06) & $(24.47)$ & (16.07) & (17.24) & $(26.20)$ & $(16.20)$ \\
\hline \multirow[t]{2}{*}{ agec } & $0.238 *$ & -0.007 & $0.248^{* *}$ & $0.246^{* *}$ & -0.006 & $0.256^{* *}$ \\
\hline & $(1.92)$ & $(-1.58)$ & $(2.01)$ & (1.99) & $(-1.38)$ & $(2.08)$ \\
\hline \multirow[t]{2}{*}{ alra } & $-0.079 * * *$ & $0.003 * * *$ & $-0.084 * * *$ & $-0.078 * * *$ & $0.003 * * *$ & $-0.084 * * *$ \\
\hline & $(-4.09)$ & $(4.01)$ & $(-4.36)$ & $(-4.07)$ & $(4.02)$ & $(-4.35)$ \\
\hline \multirow[t]{2}{*}{ ltass } & $-13.814 * * *$ & $0.198 * * *$ & $-14.114 * * *$ & $-13.702 * * *$ & $0.209 * * *$ & $-14.036 * * *$ \\
\hline & $(-21.72)$ & (12.98) & $(-21.76)$ & $(-21.72)$ & (13.74) & $(-21.77)$ \\
\hline \multirow[t]{2}{*}{ indc } & $0.627 * * *$ & $0.012 * * *$ & $0.609 * * *$ & $0.636^{* * * *}$ & $0.013 * * *$ & $0.615 * * *$ \\
\hline & $(3.21)$ & $(3.70)$ & $(3.09)$ & $(3.27)$ & $(3.82)$ & $(3.12)$ \\
\hline \multirow[t]{2}{*}{ gend } & 0.375 & 0.090 & 0.239 & 0.196 & 0.071 & 0.082 \\
\hline & $(0.22)$ & $(1.47)$ & $(0.14)$ & $(0.11)$ & $(1.16)$ & $(0.05)$ \\
\hline \multirow[t]{2}{*}{ age } & -0.062 & $0.011 * * *$ & -0.078 & -0.048 & $0.012 * * *$ & -0.067 \\
\hline & $(-0.89)$ & $(4.06)$ & $(-1.12)$ & $(-0.69)$ & $(4.55)$ & $(-0.96)$ \\
\hline \multirow[t]{2}{*}{ edu } & $1.685 * * *$ & $0.100^{* * *}$ & $1.533 * * *$ & $1.716^{* * *}$ & $0.103 * * *$ & $1.553 * * *$ \\
\hline & $(3.23)$ & $(5.39)$ & $(2.97)$ & (3.29) & $(5.49)$ & $(3.00)$ \\
\hline \multirow[t]{2}{*}{ ownp } & $-0.056 * * *$ & $-0.016 * * *$ & -0.032 & $-0.053 * * *$ & $-0.015^{* * *}$ & -0.028 \\
\hline & $(-2.87)$ & $(-21.70)$ & $(-1.60)$ & $(-2.70)$ & $(-21.22)$ & $(-1.40)$ \\
\hline \multirow[t]{2}{*}{ _cons } & $55.770 * * *$ & $1.241^{* * *}$ & $53.889 * * *$ & $53.490 * * *$ & $1.026 * * *$ & $51.854 * * *$ \\
\hline & $(9.41)$ & $(5.65)$ & $(9.07)$ & $(9.12)$ & $(4.72)$ & $(8.83)$ \\
\hline province & control & control & control & control & control & control \\
\hline industry & control & control & control & control & control & control \\
\hline year & control & control & control & control & control & control \\
\hline $\mathrm{N}$ & 7872.000 & 7872.000 & 7872.000 & 7872.000 & 7872.000 & 7872.000 \\
\hline $\mathrm{r} 2$ & 0.174 & 0.376 & 0.177 & 0.173 & 0.371 & 0.176 \\
\hline Sobel Z & & $4.233 * * *$ & & & $3.520 * * *$ & \\
\hline
\end{tabular}

Note. t statistics in parentheses, $* \mathrm{p}<0.1, * * \mathrm{p}<0.05, * * * \mathrm{p}<0.01$. 
Table 6. Impact mechanism of the tire of CPPCC and the position in CPPCC

\begin{tabular}{|c|c|c|c|c|c|c|}
\hline \multirow{2}{*}{ variables } & \multicolumn{3}{|c|}{ The tier of CPPCC } & \multicolumn{3}{|c|}{ The position in CPPCC } \\
\hline & ROA & admt & ROA & ROA & admt & ROA \\
\hline \multirow[t]{2}{*}{ cppc } & $1.305^{* * *}$ & $0.060 * * *$ & $1.209^{* *}$ & & & \\
\hline & $(2.65)$ & $(3.12)$ & $(2.47)$ & & & \\
\hline \multirow[t]{2}{*}{ admt } & & & $1.609 * * *$ & & & $1.615^{* * *}$ \\
\hline & & & $(5.08)$ & & & (5.09) \\
\hline \multirow[t]{2}{*}{ cppm } & & & & $3.699 * *$ & $0.162 * * *$ & $3.438^{* *}$ \\
\hline & & & & $(2.40)$ & $(2.58)$ & $(2.25)$ \\
\hline \multirow[t]{2}{*}{ empl } & $12.422 * * *$ & $0.536 * * *$ & $11.561 * * *$ & $12.453 * * *$ & $0.537 * * *$ & $11.586^{* * *}$ \\
\hline & $(17.22)$ & $(26.28)$ & $(16.13)$ & $(17.36)$ & $(26.37)$ & $(16.25)$ \\
\hline \multirow[t]{2}{*}{ agec } & $0.216^{*}$ & $-0.008^{*}$ & $0.228^{*}$ & $0.235^{*}$ & -0.007 & $0.246^{* *}$ \\
\hline & $(1.74)$ & $(-1.66)$ & $(1.84)$ & $(1.90)$ & $(-1.47)$ & (1.99) \\
\hline \multirow[t]{2}{*}{ alra } & $-0.077 * * *$ & $0.004 * * *$ & $-0.083^{* * *}$ & $-0.078 * * *$ & $0.003^{* * *}$ & $-0.084 * * *$ \\
\hline & $(-4.00)$ & $(4.10)$ & $(-4.29)$ & $(-4.04)$ & $(4.05)$ & $(-4.33)$ \\
\hline \multirow[t]{2}{*}{ ltass } & $-13.737 * * *$ & $0.209 * * *$ & $-14.073 * * *$ & $-13.695 * * *$ & $0.211 * * *$ & $-14.035^{* * *}$ \\
\hline & $(-21.77)$ & $(13.56)$ & $(-21.80)$ & $(-21.69)$ & (13.74) & $(-21.74)$ \\
\hline \multirow[t]{2}{*}{ indc } & $0.634 * * *$ & $0.013^{* * *}$ & $0.613^{* * *}$ & $0.635^{* * *}$ & $0.013^{* * *}$ & $0.614 * * *$ \\
\hline & $(3.25)$ & (3.73) & (3.11) & (3.27) & (3.77) & (3.13) \\
\hline \multirow[t]{2}{*}{ gend } & 0.015 & 0.061 & -0.083 & 0.041 & 0.062 & -0.060 \\
\hline & $(0.01)$ & $(1.00)$ & $(-0.05)$ & $(0.02)$ & (1.02) & $(-0.03)$ \\
\hline \multirow[t]{2}{*}{ age } & -0.043 & $0.012^{* * * *}$ & -0.062 & -0.049 & $0.012 * * *$ & -0.068 \\
\hline & $(-0.61)$ & $(4.65)$ & $(-0.89)$ & $(-0.70)$ & $(4.55)$ & $(-0.97)$ \\
\hline \multirow[t]{2}{*}{ edu } & $1.676^{* * *}$ & $0.101^{* * *}$ & $1.514 * * *$ & $1.685^{* * *}$ & $0.101^{* * *}$ & $1.522 * * *$ \\
\hline & $(3.22)$ & (5.38) & $(2.93)$ & (3.23) & $(5.40)$ & $(2.94)$ \\
\hline \multirow[t]{2}{*}{ ownp } & $-0.057 * * *$ & $-0.016^{* * *}$ & -0.032 & $-0.055 * * *$ & $-0.015^{* * *}$ & -0.030 \\
\hline & $(-2.87)$ & $(-21.41)$ & $(-1.56)$ & $(-2.81)$ & $(-21.32)$ & $(-1.50)$ \\
\hline \multirow[t]{2}{*}{ _cons } & $53.361 * * *$ & $1.008 * * *$ & $51.741 * * *$ & $53.381 * * *$ & $1.007 * * *$ & $51.754 * * *$ \\
\hline & (9.11) & (4.63) & (8.82) & (9.12) & (4.62) & (8.83) \\
\hline province & control & control & control & control & control & control \\
\hline industry & control & control & control & control & control & control \\
\hline year & control & control & control & control & control & control \\
\hline $\mathrm{N}$ & 7872.000 & 7872.000 & 7872.000 & 7872.000 & 7872.000 & 7872.000 \\
\hline $\mathrm{r} 2$ & 0.173 & 0.370 & 0.176 & 0.173 & 0.369 & 0.176 \\
\hline Sobel Z & & $2.660 * *$ & & & $2.299 * * *$ & \\
\hline
\end{tabular}

Note. $\mathrm{t}$ statistics in parentheses, ${ }^{*} \mathrm{p}<0.1, * * \mathrm{p}<0.05$, *** $\mathrm{p}<0.01$.

Table 7. Impact mechanism of the tire of the position in government institutions and state-owned or collective-owned enterprises

\begin{tabular}{|c|c|c|c|c|c|c|}
\hline \multirow{2}{*}{ variables } & \multicolumn{3}{|c|}{ The position in government institutions } & \multicolumn{3}{|c|}{ The position in state-owned or collective-owned enterprises } \\
\hline & ROA & admt & ROA & ROA & admt & ROA \\
\hline parg & $\begin{array}{c}1.862 * * \\
(2.14)\end{array}$ & $\begin{array}{c}0.129 * * * \\
(3.93)\end{array}$ & $\begin{array}{l}1.655^{*} \\
(1.92)\end{array}$ & & & \\
\hline admt & & & $\begin{array}{c}1.610^{* * * *} \\
(5.08)\end{array}$ & & & $\begin{array}{c}1.544 * * * \\
(4.81)\end{array}$ \\
\hline esta & & & & $\begin{array}{c}1.067 * * * \\
(3.93)\end{array}$ & $\begin{array}{c}0.081 * * * \\
(7.67)\end{array}$ & $\begin{array}{c}0.941 * * * \\
(3.43)\end{array}$ \\
\hline empl & $\begin{array}{c}12.494 * * * \\
(17.35)\end{array}$ & $\begin{array}{c}0.538^{* * * *} \\
(26.47)\end{array}$ & $\begin{array}{c}11.628^{* * * *} \\
(16.26)\end{array}$ & $\begin{array}{c}12.458^{* * * *} \\
(17.27)\end{array}$ & $\begin{array}{c}0.535 * * * \\
(26.39)\end{array}$ & $\begin{array}{c}11.632 * * * \\
(16.27)\end{array}$ \\
\hline agec & $\begin{array}{c}0.260 * * \\
(2.10)\end{array}$ & $\begin{array}{l}-0.005 \\
(-1.18)\end{array}$ & $\begin{array}{c}0.268 * * \\
(2.17)\end{array}$ & $\begin{array}{c}0.263 * * \\
(2.13)\end{array}$ & $\begin{array}{l}-0.005 \\
(-1.10)\end{array}$ & $\begin{array}{c}0.271 * * \\
(2.20)\end{array}$ \\
\hline alra & $\begin{array}{c}-0.078 * * * \\
(-4.04)\end{array}$ & $\begin{array}{c}0.003^{* * *} * \\
(4.06)\end{array}$ & $\begin{array}{c}-0.084 * * * \\
(-4.34)\end{array}$ & $\begin{array}{c}-0.080 * * * \\
(-4.14)\end{array}$ & $\begin{array}{c}0.003 * * * \\
(3.88)\end{array}$ & $\begin{array}{c}-0.085^{* * *} \\
(-4.41)\end{array}$ \\
\hline ltass & $\begin{array}{c}-13.660 * * * \\
(-21.71)\end{array}$ & $\begin{array}{c}0.212^{* * * *} \\
(13.87)\end{array}$ & $\begin{array}{c}-14.001 * * * \\
(-21.76)\end{array}$ & $\begin{array}{c}-13.648^{* * *} \\
(-21.72)\end{array}$ & $\begin{array}{c}0.212 * * * \\
(13.93)\end{array}$ & $\begin{array}{c}-13.976^{* * *} \\
(-21.71)\end{array}$ \\
\hline indc & $\begin{array}{c}0.636^{* * * *} \\
(3.28)\end{array}$ & $\begin{array}{c}0.013^{* * * *} \\
(3.81)\end{array}$ & $\begin{array}{c}0.615^{* * * *} \\
(3.13)\end{array}$ & $\begin{array}{c}0.635 * * * \\
(3.26)\end{array}$ & $\begin{array}{c}0.013 * * * \\
(3.75)\end{array}$ & $\begin{array}{c}0.615^{* * *} * \\
(3.12)\end{array}$ \\
\hline
\end{tabular}




\begin{tabular}{|c|c|c|c|c|c|c|}
\hline \multirow[t]{2}{*}{ gend } & 0.011 & 0.059 & -0.084 & -0.239 & 0.040 & -0.301 \\
\hline & $(0.01)$ & $(0.97)$ & $(-0.05)$ & $(-0.14)$ & $(0.65)$ & $(-0.17)$ \\
\hline \multirow[t]{2}{*}{ age } & -0.062 & $0.011 * * *$ & -0.080 & -0.106 & $0.007 * * *$ & -0.118 \\
\hline & $(-0.89)$ & $(4.14)$ & $(-1.14)$ & $(-1.48)$ & $(2.77)$ & $(-1.64)$ \\
\hline \multirow[t]{2}{*}{ edu } & $1.626^{* * *}$ & $0.097 * * *$ & $1.471 * * *$ & $1.535 * * *$ & $0.089 * * *$ & $1.398 * * *$ \\
\hline & $(3.10)$ & $(5.15)$ & $(2.83)$ & $(2.93)$ & $(4.76)$ & (2.69) \\
\hline \multirow[t]{2}{*}{ ownp } & $-0.051 * *$ & $-0.015 * * *$ & -0.026 & $-0.044 * *$ & $-0.015^{* * *}$ & -0.022 \\
\hline & $(-2.58)$ & $(-20.95)$ & $(-1.29)$ & $(-2.22)$ & $(-20.28)$ & $(-1.05)$ \\
\hline \multirow{2}{*}{ _cons } & $53.119 * * *$ & $1.005 * * *$ & $51.501 * * *$ & $54.026 * * *$ & $1.077 * * *$ & $52.363 * * *$ \\
\hline & (9.04) & $(4.62)$ & $(8.75)$ & $(9.24)$ & (4.98) & $(8.94)$ \\
\hline province & control & control & control & control & control & control \\
\hline industry & control & control & control & control & control & control \\
\hline year & control & control & control & control & control & control \\
\hline $\mathrm{N}$ & 7872.000 & 7872.000 & 7872.000 & 7872.000 & 7872.000 & 7872.000 \\
\hline $\mathrm{r} 2$ & 0.173 & 0.370 & 0.176 & 0.174 & 0.374 & 0.177 \\
\hline Sobel Z & & $3.107 * * *$ & & & $4.078 * * *$ & \\
\hline
\end{tabular}

Note. $\mathrm{t}$ statistics in parentheses, $* \mathrm{p}<0.1, * * \mathrm{p}<0.05, * * * \mathrm{p}<0.01$.

Table 5, Table 6 and Table 7 are empirical results of different tiers of political connections' influence on private enterprise performance. With regard to current political connections, Table 5 shows that the tier of NPC is positive to both enterprise performance and enterprise internal governance. When simultaneously adding the tier of NPC and enterprise internal governance into regression, both the variables are still positive to enterprise performance, which illustrates that current political connections like the tier of NPC can ameliorate enterprise performance through enterprise internal governance. Therefore, enterprise internal governance plays as mediating factor and accounts for $13.1 \%$ of total effects. Likewise, the mediating effects of internal governance also exist in the relationship between other current political connections and enterprise performance, including the position in NPC (10\%), the tier of CPPCC (7.4\%), the position in CPPCC (7.1\%), the position in government institutions (11.2\%) and the position in state-owned or collective-owned enterprises (11.7\%). In a word, enterprise internal governance is the mediating factor to improve enterprise performance. The results support Hypothesis $4 \mathrm{a}$.

\section{Conclusions}

According to previous analysis, two main conclusions are drawn: (1) Private enterprises with political connections perform better. Specifically, different kinds or tires of political connections all ameliorate private enterprise performance while current political connections impose better effects on enterprise performance compared with former political connections; (2) Enterprise internal governance plays as mediating role in the relationship between political connections and private enterprise performance, which is also true to different kinds of political connections.

The empirical results in the paper also shed light on private enterprise development in China in practice. First, government should streamline administration and delegate more power to lower-level governments, and establish more friendly environment to spur vitality of private enterprises. Second, private enterprises should rationally build their own political connections. Meanwhile, this paper implies that in a special market environment which traditionally supports state-owned enterprises in China, private entrepreneurs taking part in politics or employees leaving state-owned enterprises and government institutions to start their own business can correct the tendency of investing too much to state-owned enterprises to some extent. This phenomenon happens to be consistent with China's recent policy to build a new type of cordial and clean relationship between government and business.

There are two limitaions of the study.One is the sample selection has certain limitations. Although the CPES data used in this article can represent most common Chinese private enterprises, it is limited to the author's ability and the work discipline requirements of the data-providing agency, and only the cross-sectional data of the relevant year can be obtained, which restricts the analysis of this article. In the future we will expand the sample and enrich the research level.

Another limitations is the measurement of variables. In this paper, only financial indicators based on the return on assets ratio are selected, and indicators such as operation, market and social indicators are not considered. As the corporate performance system continues to improve, scholars are also paying more and more attention to the combination of quantitative evaluations in finance, market, and operations with qualitative evaluations in 
management and society. Therefore, in the future, it is also possible to measure corporate performance from multiple angles based on measurement methods such as market performance and comprehensive performance.

\section{References}

Ba, S. S., \& Zhu, W. H. (2017). Ownership, political relations and taxation burden. Journal of Financial Development Research, 8, 3-14.

Boubakri, N., Guedhami, O., \& Mishra, D. (2008). Political connections and the cost of equity capital. Journal of Corporate Finance, 18(3), 541-559. https://doi.org/10.2139/ssrn.1589688

Chaney. (2011). Theory of the firm: Managerial behavior, agency costs and ownership structure. Journal of Financial Economic, 5(3), 305-312.

Cull, R., \& Xu, L. C. (2005). Institutions, ownership, and finance: The determinants of profit reinvestment among Chinese firms. Journal of Financial Economics, 77(1), 117-146. https://doi.org/10.1016/j.jfineco.2004.05.010

Faccio, M. (2006). Politically connected firms. American Economic Eview, 96(1), 369-386. https://doi.org/10.1257/000282806776157704

Fan, J. P. H., Wong, T. J., \& Zhang, T. (2007). Politically connected CEOs, corporate governance, and post-IPO performance of China's newly partially privatized firms. Journal of Financial Economics, 84(2), 330-357. https://doi.org/10.1016/j.jfineco.2006.03.008

Galal, \& Mgeginsno. (1998). Ownership structure, managerial behavior and corporate value. Journal of Finance, 11(4), 645-660. https://doi.org/10.1016/j.jcorpfin.2004.07.001

He, X. G., Zhang, Y. F., \& Lian, Y. L. (2019). Political ties and firm value-An empirical study in China. China Industrial Economics, 1, 103-115.

Lei, G. Y., Li, S. F., \& Wang, X. J. (2009). Political ties, auditor choice and enterprise value. Management World, 7, 145-155.

Li, K. Y., Gao, Y., \& Wu, C. (2013). Political relations of the entrepreneurs and degree of the market which for their companies to enter the dministrative monopoly industries - An empirical study based on China's private enterprises. Research on Economics and Management, 9, 95-104.

Luo, D. L., \& Huang, Q. Y. (2008). Political relationship and firm value of private company. Journal of Management Sciences, 6(21), 28.

Niessen, A., \& Ruenzi, S. (2010). Political connectedness and firm performance: Evidence from germay. German Economic Review, 11(4), 441-464. https://doi.org/10.1111/j.1468-0475.2009.00482.x

Wu, W. F., Wu, C. F., \& Liu, X. W. (2008). Political connection and market valuation: Evidence from China individual-controlled listed firms economic research. Economic Research Journal, 7,130-141.

Yang, H. Y., \& Yang, J. (2020). Influence of environmental regulation on enterprise performance: An empirical study based on enterprise social responsibility. Journal of Shandong University of Finance and Economics, 2, 99-107.

Yang, J., \& Zhang, P. (2018). Local legal environment and firm performance__ An empirical research based on the survey data of business environment in China. Journal of Shanxi University of Finance and Economics, $9,1-17$.

Zhang, X. J., \& Guo, L. (2010). Mechanism, path and strategy of political connections. Finance \& Trade Economics, 9, 99-104.

Zhou, W. (2009). Bank financing in china's private sector: The payoffs of political capital. World Development, 37(4), 787-799. https://doi.org/10.1016/j.worlddev.2008.07.011

\section{Copyrights}

Copyright for this article is retained by the author(s), with first publication rights granted to the journal.

This is an open-access article distributed under the terms and conditions of the Creative Commons Attribution license (http://creativecommons.org/licenses/by/4.0/). 